\title{
Simulation of Corticospinal Tract Displacement in Patients with Brain Tumors
}

\author{
M.R. Kaus ${ }^{1}$, A. Nabavi ${ }^{2,3}$, C.T. Mamisch ${ }^{2}$, W.H. Wells ${ }^{2,4}$, F.A. Jolesz ${ }^{2}$,
} R. Kikinis ${ }^{2}$, and S.K. Warfield ${ }^{2}$

1 Philips Research Laboratories, Division Technical Systems, Röntgenstraße 24-26, 22335 Hamburg, Germany, M.Kaus@pfh.research.philips.com

2 Surgical Planning Laboratory, Department of Radiology

3 Department of Neurosurgery, Brigham \& Womens Hospital, Harvard Medical School, 75 Francis Street, Boston, MA 02115

4 Artificial Intelligence Lab, MIT, 545 Technology Square, Cambridge, MA 02139

\begin{abstract}
The spatial relationship between the corticospinal tracts and a brain tumor is important for planning the surgical strategy. Although the white matter tracts can be manually outlined from structural MRI this is time consuming and impractical. To enhance the spatial understanding of the distorted pathology we have established a method to retrieve this structural information automatically by registration of a standardized normal brain atlas to the individual pathologic anatomy of brain tumor patients. The skin and the brain were segmented in the patient MRI volume. Subsequently a deformable volumetric atlas of a single normal subject was registered to the patient brain using affine and non-linear registration techniques. The estimated spatial correspondence between atlas and patient brain was used to warp the corticospinal tracts from the atlas onto the patient. The accuracy of the method was evaluated in 5 patients with extrinsic tumors of different histopathology and location by comparing selected anatomical landmark structures from the projected atlas to their manually segmented counterpart in the patient. Our method enables the visualization of complex anatomical information with minimal user interaction for routine surgical planning that would otherwise demand the acquisition of additional imaging modalities.
\end{abstract}

\section{Introduction}

Computer assisted surgical planning aids the neurosurgeon in the appreciation of the spatial arrangement of critical brain structures (e.g. motor and sensory cortex) with respect to an adjacent lesion, to define the safest possible surgical approach. If a tumor is located close to the motor cortex, the location of the corticospinal tracts (CST, Figure 2) is particularly important, but unproportionally more difficult to visualize. Nevertheless, connecting functional areas, damage to these white matter tracts results in the same clinical deficits as the destruction of those centers themselves. 
Although diffusion tensor MRI provides direct visual information of the white matter tracts, this imaging technique and related image post-processing algorithms are not yet at the stage of providing routinely available studies $[1 / 23]$. Manual segmentation of the corticospinal tracts from conventional MRI is difficult if not impossible. Even an experienced, trained radiologist can hardly distinguish different tracts from the bulk of the white matter. In addition, manual segmentation would be time consuming, excluding this approach from clinical routine. Automated segmentation techniques based on image features alone are also inadequate for this task, because the different tracts are almost indistinguishable on conventional MRI.

Non-linear registration of a deformable anatomical template has been used for various image analysis tasks where image information alone is not sufficient. Applications include the identification of cortical sulci or functional areas, and the segmentation of multiple sclerosis lesions or brain tumors $4,5,6,7,8$. Early work of this group reported the volume measurement of basal ganglia schizophrenics by registration of a digital atlas 9 . However, no work has been reported on using an anatomical atlas to retrieve structural information in brain tumor patients where dramatic brain deformation can occur.

In this work we present an automated method which is based on [9] with some variations to retrieve structural information in patients with brain tumors using a standardized normal brain atlas. As an example for a clinically relevant structure, we use our method to extract the corticospinal tracts in 5 patients and validate the results with anatomical landmark structures semi-automatically segmented by trained clinical personnel in MRI and fMRI.

\section{Methods}

We used a multistage image registration scheme (Figure 10 to align a 3D template of normal anatomy (a deformable volumetric digital brain atlas) to identify structural information in 3D MRI of brain tumor patients. A hierarchical strategy was implemented to avoid local minima in the spatial transform parameter search space and to capture large deformations that can be caused by a brain tumor. The spatial transform that maps the atlas brain onto the patient brain was used to warp the structures of interest (i.e. the corticospinal tracts) from the atlas onto the individual patient dataset. In the following sections we will describe the image data used, the image processing steps, and the validation procedure.

\subsection{Image Data}

\section{Atlas Data}

We used a volumetric brain atlas based of a single normal male where each voxel was labeled (given a number) according to its anatomical membership [10]. For the purpose of hierarchical registration, separate template volumes that contain only one structure (e.g. all segmented structures to form the head volume) were 
extracted. Here the head volume, the brain volume and the structural volume (containing voxels labeled as thalamus, nucleus caudatus, globus pallidus, preand postcentral gyrus, and corticospinal tracts) were formed. The structural volume was projected for validation purposes (see Section 2.3).

\section{Patient Data}

5 patients were scanned with a standard protocol (1.5 T Signa GE MRI, postcontrast $3 \mathrm{D}$ sagittal spoiled gradient recalled (SPGR) acquisition, $256 \times 256$ $\times 124,0.9375 \times 0.9375 \times 1.5 \mathrm{~mm}^{3}$ ). For one patient (no. 5), a standard fMRI sequence was also available for visualization of the motor cortex. The head, brain and structural volume were semi-automatically segmented in the MRI using a 3D segmentation tool [1].

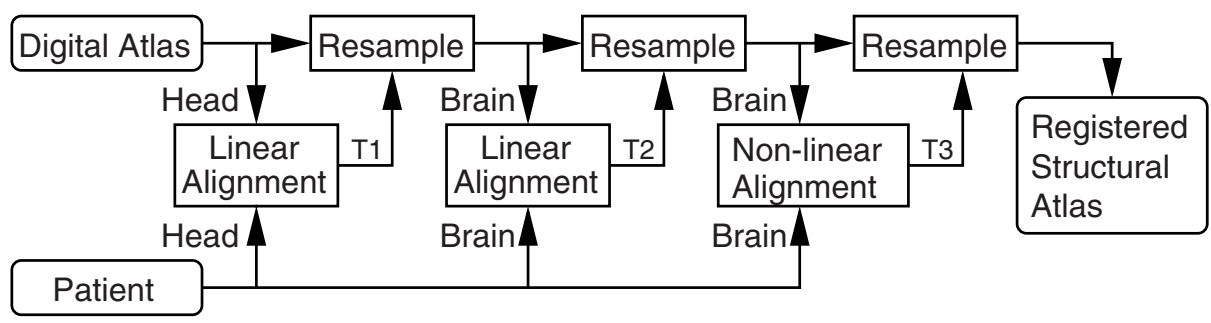

Fig. 1. Schematic description of the registration procedure. Global shape differences are captured with the global affine transforms $T_{1}$ and $T_{2}$, while local shape differences are represented with a volumetric deformation vector field $T_{3}$.

\subsection{Registration Procedure}

Linear Registration The global affine transform $T_{1}: I_{1}(\mathbf{x}) \rightarrow I_{2}(\mathbf{x})$, that roughly aligned the atlas head volume $I_{1}(\mathbf{x})$ to the patient $I_{2}(\mathbf{x})$ where $\mathbf{x}=$ $[x, y, z]^{T}$ was estimated in a 2 -steps procedure. First, an initial pose was estimated by aligning the back and the top of the atlas and the patient heads bounding boxes. Second, using a linear registration method for segmented images [12], the head of the atlas and the patient were registered by minimizing

$$
S=\sum_{i=1}^{N} f\left(I_{2}\left(\mathbf{x}_{i}\right), I_{1}\left(T^{-1}\left(\mathbf{x}_{i}\right)\right)\right.
$$

using Powells method, where $N$ is the total number of voxels and $f(a, b)=0$ if $a=b$ and $f(a, b)=1$ otherwise. For binary data $f$ could be efficiently computed using the xor function. To decrease conversion time, a multiresolution image pyramid was formed, subsampling the images by a factor of 2 in each spatial 
dimension at every resolution level. The minimization was carried from coarseto-fine resolution on three levels.

The atlas was resampled according to $T_{1}$ using nearest neighbor interpolation, the brain volume was extracted from the resampled atlas, and the 2 -step linear alignment procedure described above was repeated to estimate $T_{2}$ to globally align the atlas and the patient brain. It was found experimentally that brains were aligned more precisely when preceded with a global affine head registration.

\section{Non-linear Registration}

\section{D Adaptive Template Matching}

We used 3D adaptive template matching to estimate a 3D volumetric deformation vector field based on the work of Dengler et al. 13. Region-based template matching defines a relative translation $\mathbf{u}(\mathbf{x})=[u(\mathbf{x}), v(\mathbf{x}), w(\mathbf{x})]^{T}$ between a voxel $\mathbf{x}$ in the atlas volume $I_{1}(\mathbf{x})$ and the patient volume $I_{2}(\mathbf{x})$. The best match is found by minimizing

$$
\mathbf{S}(\mathbf{u}(\mathbf{x}))=\int_{I_{2}} W(\mathbf{x})\left(I_{2}(\mathbf{x})-I_{1}(\mathbf{x}-\mathbf{u}(\mathbf{x}))\right)^{2} d \mathbf{x} .
$$

$W$ is a window function whose width controls the size of the image patches that are compared. Deformations are assumed to be constant in this region and only estimated locally, such that $S$ is effectively a function of $\mathbf{x}$. Linearizing $I_{1}(\mathbf{x}-\mathbf{u}(\mathbf{x})) \approx I_{1}(\mathbf{x})-\mathbf{u}(\mathbf{x}) \nabla I_{1}(\mathbf{x})$ allows a closed form solution of Equation 2 for each voxel. Setting $\mathbf{S}(\mathbf{u}) / d \mathbf{u}=0$ leads to

$$
\mathbf{Q u}=-\mathbf{f}, \quad \mathbf{Q}=\int_{I_{2}} W \nabla I_{1} \nabla I_{1}^{T} d \mathbf{x}, \quad \mathbf{f}=\int_{I_{2}} W \nabla I_{1}\left(I_{2}-I_{1}\right) d \mathbf{x}
$$

which can be directly solved with Cramers rule (dependence on $\mathbf{x}$ omitted for clarity).

Linearization assumes small $\mathbf{u}$. To justify this assumption, the atlas is linearly registered (Section 2.2) to the patient prior to the non-linear registration, and the deformation vector field is estimated on a coarse-to-fine resolution pyramid. Gaussian smoothing and subsampling is efficiently calculated according to Burt et al. 14. Because $\nabla I_{1}$ is not defined for segmented data, $I_{1}$ (and $I_{2}$ ) were smoothed with a 5 point Gaussian prior to the computation of the derivates.

In regions where the gradient $\nabla I$ is close or equal to $0, \mathbf{Q}$ is not invertible and Equation 3 has no solution, i.e. the deformation cannot be estimated in unstructured regions. Therefore, $W$ needs to be large enough to allow computation of $\nabla I_{1}$ and enable reliable estimation of $\mathbf{u}$ respectively. Because $W$ also determines the variability of $\mathbf{u}$ over the image volume, and we want to allow $\mathbf{u}$ to vary rapidly where necessary, $W$ should be as small as possible and as large as necessary. Thus, we use a spatially varying adaptive window function $W$

$$
W=W(\mathbf{x})=\sum_{i=1}^{i_{\max }} w_{i} W_{i} \quad, \quad w_{i}=\frac{1}{\sum_{i} \operatorname{det}\left(\mathbf{Q}_{i}\right)} \operatorname{det}\left(\mathbf{Q}_{i}\right) \quad,
$$


that takes into account several window functions $W_{i}$ of size $s_{i+1}=2 s_{i}$, where $s_{i}$ is limited by the smallest volume axis length. Qualitatively, the weight $w_{i}$ is large if the image patch contains large image gradients in a variety of directions. If a small image patch is sufficiently structured then $\mathbf{u}$ can be estimated with a small patch, otherwise larger image patches dominate the estimation. If $\operatorname{det} \mathbf{Q}_{i}(\mathbf{x})=0$, $\forall i, \mathbf{u}(\mathbf{x})$ is set to zero.

Our template matching approach results in a smooth deformation field $T_{3}$. Since neighboring voxels have similar neighborhoods, the similarity measure (integrated over the window patches) at neighboring voxels is also similar, resulting in a slowly varying or smooth deformation field.

\section{Image Warping}

Ideally, to construct the deformed template image $\tilde{I}_{1} \leftarrow I_{1}$, $\mathbf{u}$ is used to translate the value at each location in $I_{1}$ according to

$$
\tilde{I}_{1}(\mathbf{x})=I_{1}(\mathbf{x}-\mathbf{u}(\mathbf{x}))=I_{1}(\tilde{\mathbf{x}}) .
$$

However, our method does not guarantee a one-to-one mapping. In case of e.g. a one-to-many mapping, this resampling method could lead to holes in the deformed template image. Therefore, we estimate the corresponding voxel position in the template image for each voxel in the target image, which essentially means to estimate the inverse of the deformation field $\mathbf{u}(\mathbf{x})$. Assuming that the deformation field is slowly varying, the necessary inverse transform can be approximated with

$$
\mathbf{u}(\tilde{\mathbf{x}})^{-1} \approx-\mathbf{u}(\mathbf{x}) .
$$

Since $\tilde{\mathbf{x}}$ in general will not be a point on the regular grid, $I_{1}(\tilde{\mathbf{x}})$ is assigned the value $I_{1}(\overline{\mathbf{x}}), \overline{\mathbf{x}}$ being the closest point to $\tilde{\mathbf{x}}$ (nearest neighbor interpolation). This kind of interpolation has the advantage of computational efficiency (as opposed to e.g. tri-linear interpolation) and maintains the original voxel values, which is advantagous when operating on segmented data.

\subsection{Validation Experiment}

We applied the method to 5 patients with meningiomas and subdural low grade gliomas. Direct validation would be possible by comparing our results to diffusion weighted images. But for the reasons mentioned above their availability is limited. Therefore we chose the approach to a) measure how well our method registers the atlas to the patient brain and b) test the anatomically correct course of the simulated white matter tracts (Figure 2). We validated the CST projections by comparing anatomical landmarks (structures in the brain) which interface with the CST and are unequivocally distinguishable and can thus be segmented from MRI. Such structures are the thalamus, the nucleus caudatus, the globus pallidus, and the pre- and postcentral gyrus. If these control structures from the warped atlas match their manually segmented counterparts, then we infer that the CST are projected correctly. 
Table 1. Similarity between manually segmented and projected control structures with respect to the volume of the bounding box. Reasonable accuracy was achieved for all structures except for the globus pallidus.

\begin{tabular}{|c|c|c|c|c|c|c|c|c|}
\hline \multirow{2}{*}{ Location } & \multirow{2}{*}{ Structure } & \multicolumn{5}{|c|}{ Case No. (Tumor location) } & \multirow{2}{*}{ Mean } & \multirow{2}{*}{$\mathrm{SD}$} \\
\hline & & $1(\mathrm{~L})$ & $2(\mathrm{~L})$ & $3(\mathrm{R})$ & $4(\mathrm{R})$ & $5(\mathrm{~L})$ & & \\
\hline \multirow{2}{*}{ Left \& Right } & Brain & 92.64 & 92.29 & 90.95 & 92.34 & 92.81 & 92.21 & 0.73 \\
\hline & Thalamus & 79.46 & 83.35 & 68.24 & 75.88 & 74.30 & 76.25 & 5.68 \\
\hline \multirow{4}{*}{ Left } & Nucleus Caudate & 92.71 & 87.12 & 76.30 & 86.94 & 88.22 & 86.26 & 6.04 \\
\hline & Globus Pallidus & 45.28 & 42.32 & 45.50 & 42.75 & 46.66 & 44.50 & 1.88 \\
\hline & Precentral Gyri & 79.58 & 79.53 & 77.81 & 77.30 & 93.87 & 81.62 & 6.92 \\
\hline & Postcentral Gyri & 75.63 & 83.78 & 74.06 & 80.18 & 91.29 & 80.98 & 6.92 \\
\hline \multirow{4}{*}{ Right } & Nucleus Caudate & 88.28 & 86.06 & 76.00 & 86.01 & 90.43 & 85.36 & 5.54 \\
\hline & Globus Pallidus & 45.70 & 42.48 & 44.39 & 41.24 & 39.05 & 42.57 & 2.61 \\
\hline & Precentral Gyri & 82.70 & 80.82 & 77.90 & 78.25 & 88.76 & 81.87 & 4.42 \\
\hline & Postcentral Gyri & 69.90 & 82.86 & 76.12 & 83.34 & 89.25 & 80.29 & 7.44 \\
\hline
\end{tabular}

The similarity $S_{s}$ between a manually segmented and a warped structure $s$ was defined as

$$
S_{s}=\frac{\#_{B_{s}} \text { OverlappingVoxels }}{\#_{B_{s}} \text { Voxels }},
$$

where \# is the counting operator applied inside the bounding box $B_{s}$ that encloses both the manually segmented and the warped control structure. The bounding box was introduced to prevent the similarity measure to be biased towards small structures.

\section{Results}

Although the brain was significantly deformed by the meningioma, visual validation of the projected corticospinal tracts (CST, Figure 2) correlated well with the anatomical definition. The CST started at the motor cortex (manually segmented from fMRI), passed through the internal capsule (between thalamus and globus pallidus, manually segmented from MRI) and lead into the crus cerebri (part of the brainstem, MRI slice). Thus origin and course towards the brainstem were simulated correctly.

In this image, the size of the motor cortex from fMRI is smaller than the corticospinal tract. This is possibly due to the fact that only a part of the motor cortex has been activated during fMRI.

Table 1 shows the similarity between the manually segmented and the projected structures. The accuracy of the brain match is the accuracy with which 


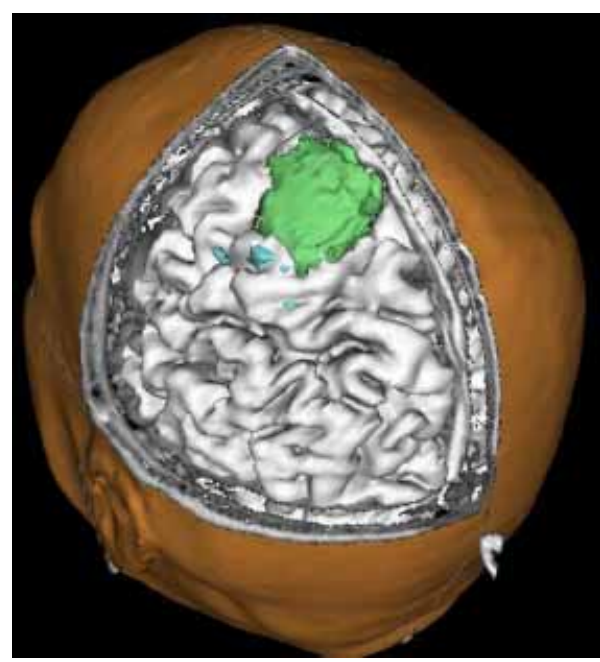

(a) Brain (White) and Tumor (Green) from MRI

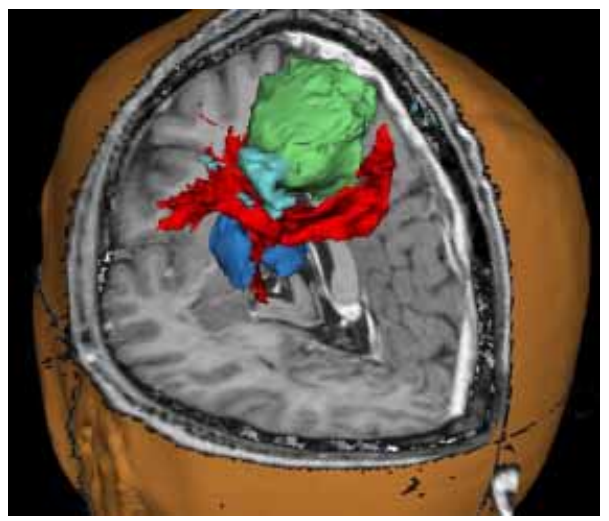

(c) Basal Ganglia (Dark Blue)

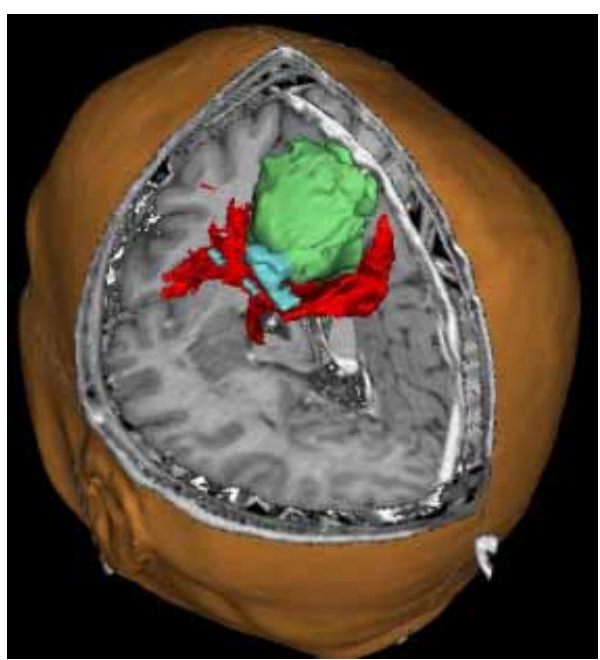

(b) Corticospinal Tract (Red) and Motor Cortex from fMRI (Light Blue)

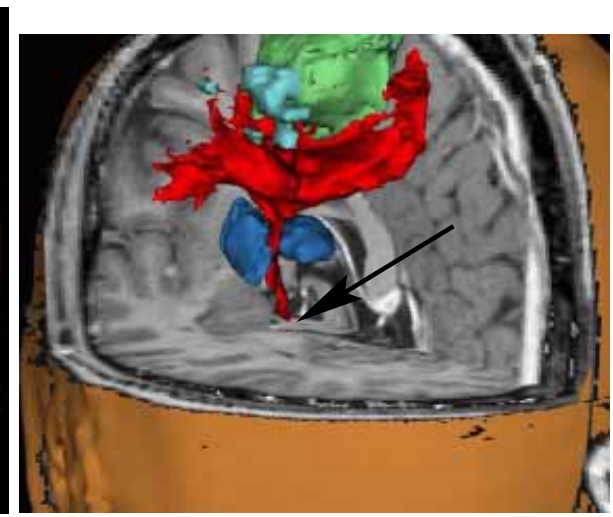

(d) Crus Cerebri (Arrow)

Fig. 2. Different views (patient no. 5) for visual verification of the corticospinal tract projection. Despite a significant brain deformation caused by the meningioma, the corticospinal tract (registered atlas) correctly connects the motor cortex (manually segmented from fMRI, (b)) to the crus cerebri (MRI slice, (c)) and runs between the globus pallidus and the thalamus (manually segmented from MRI, (d)). 
our method maps one structure onto another by means of minimization of a similarity measure. The accuracy of the landmark structure registration describes how well our model describes the brain distortion caused by the pathological process.

As expected, the brains matched better than the control structures. The reason is that the correspondence between the atlas and the patient is established by registration of the atlas to the patient brain. The mapping of the control structures is based on our model of brain deformation. A deformation field based on image structure is only estimated on the the brain surface and the brain-ventricle interface, while the deformation field resulting for the inner brain structures are purely based on interpolation. However, reasonable similarity was established for all control structures. The globus pallidus was aligned with less accuracy, which can be partly explained with an oversegmented globus pallidus in our anatomical atlas.

\section{Discussion and Conclusion}

We have developed a method for the automated extraction of non-visible inherent information of a structural scan based on non-linear template matching of a brain atlas. It was demonstrated that structural information can be projected from a standardized brain atlas from a normal single subject onto patients with brain tumors with reasonable accuracy. This enables the visualization of complex anatomical information for surgical planning that would otherwise demand the acquisition of additional imaging modalities.

The white matter tracts have only recently been visualized in vivo employing diffusion weighted images 213. Although their anatomical course has been extensively studied, the surgical anatomy in pathologically deformed brain could not be described presurgically. Since the advent of image guided surgery 3D representation of the anatomy and the co-registration of functional data 16 has increased the interest in precise surgical planning, particularly for tumors adjacent to eloquent areas. The incorporation of functional data has enabled the surgical approach to these lesions. Nevertheless a remaining problem is the displacement of the subcortical fiber connections. The unequivocal identification of functional areas is reported to significantly improve approaches and potential outcome of patients harboring lesions in these areas [16]. Nevertheless it is apparent, that keeping cortical areas intact, while disregarding their connections will result in the same deleterious clinical result, as damaging those cortical areas themselves. Therefore it is of great importance to include the connecting tracts, and particularly the corticospinal tracts.

Particularly the minimal user interaction as well as the utilization of information already available from conventional planning and neuronavigation enhances the potential use of this method. In this paper we used initial semi-automated segmentation, as needed for surgical planning. However, automated methods have become available potentially decreasing user interaction even for this step [15]. 
Several questions for future work remain. Our method is currently limited to extrinsic tumors because our template matching approach requires equivalent topology. This is not the case when mapping a normal brain atlas to a brain with an intrinsic tumor. However, recent work on atlas deformation in the presence of intrinsic tumors could be investigated in this context [17. We are currently working on a more solid validation study based on intraoperative verification and preferably on tensor diffusion MRI with a larger number of cases.

\section{Acknowledgments}

This investigation was supported (in part) by a Grant from the National Multiple Sclerosis Society (SW). This work was supported (in part) by NIH grants RO1 CA 46627-08, PO1 CA67165-01A1, PO1 AG04953-14, NSF grant BES 9631710, Darpa grant F41624-96-2-0001, Whittaker Foundation RG-96-0440, and DFG (NA 359/1-1).

\section{References}

1. C. Pierpaoli, P. Jezzard, P.J. Basser, A. Barnett, and G. Di Chiro. Diffusion tensor MR imaging of the human brain. Radiology, 201:637-648, 1996.

2. C.F. Westin, S.E. Maier, B. Khidhir, P. Everett, F.A. Jolesz, and R. Kikinis. Image processing for diffusion tensor MRI. In MICCAI 99, pages 441-452, 1999.

3. C. Poupon, J.F. Mangin, V. Frouin, M. Pachot-Clouard, D. Le Bihain and I. Bloch. Regularization of MR diffusion tensor maps for tracking brain white matter bundles. In MICCAI 98, pages 489-498, 1998.

4. R. Bajcsy, R. Lieberson, and M. Reivich. A computerized system for the elastic matching of deformable radiographic images to idealized atlas images. JCAT, 7(4):618-625, 1983.

5. G.E. Christensen, R.D. Rabbitt, and M.I. Miller. 3D brain mapping using a deformable neuronatomy. Physics in Medicine and Biology, 39:609-618, 1994.

6. P. Thompson, D. MacDonald, M.S. Mega, C.J. Holmes, A. Evans, and A.W. Toga. Detection and mapping of abnormal brain structures with a probabilistic atlas of cortical surfaces. JCAT, 21(4):467-481, 1996.

7. D.L. Collins, T.M. Peters, W. Dai, and A.C. Evans. Model based segmentation of individual brain structures from MRI data. In VBC 92, pages 10-23, 1992.

8. S.K. Warfield, M.R. Kaus, F.A. Jolesz, and R. Kikinis. Adaptive template moderated spatially varying statistical classification. In MICCAI 98, Boston, MA, USA, 1998.

9. D.V. Iosifescu, M.E. Shenton, S.K. Warfield, R. Kikinis, J. Dengler, F.A. Jolesz, and R.W. McCarley. An automated measurement of subcortical brain MR structures in schizophrenia. Neuroimage, 6:13-25, 1997.

10. R. Kikinis, M.E. Shenton, D.V. Iosifescu, R.W. McCarley, P. Saiviroonporn, H.H. Hokama, A. Robatino, D. Metcalf, C.G. Wible, C.M. Portas, R. Donnino and F.A. Jolesz. A digital brain atlas for surgical planning, model driven segmentation and teaching. IEEE VCG, 2(3):232-241, 1996. 
11. D.T Gering, A. Nabavi, R. Kikinis, W.E.L. Grimson, N. Hata, P. Everett, F.A. Jolesz and W.M. Wells. An integrated visualization system for surgical planning and guidance using image fusion and interventional imaging. In MICCAI 99, pages 809-819, Cambridge, UK, 1999.

12. S.K. Warfield, F.A. Jolesz, and R. Kikinis. A high performance computing approach to the registration of medical image data. Parallel Computing, 24:13451368, 1998.

13. J. Dengler and M. Schmidt. The dynamic pyramid - a model for motion analysis with controlled continuity. PRAI, 2(2):275-286, 1987.

14. P.J. Burt and E.H. Adelson. The laplacian pyramid as a compact image code. IEEE Transactions on Communications, 31(4):532-510, 1983.

15. M.R. Kaus, S.K. Warfield, A. Nabavi, E. Chatzidakis, P. Black, F.A. Jolesz and R. Kikinis. Automated brain tumor segmentation in MRI: meningiomas and low grade gliomas. In MICCAI 99, pages 1-10, 1999.

16. O. Ganslandt, R. Fahlbusch, C. Nimsky, H. Kober, M. Müller, R. Steinmeier, and J. Romstöck. Functional neuronavigation with MEG: outcome in 50 patients with lesions around the motor cortex. Neurosurg Focus, 6(3):Article 3, 1999.

17. B.M. Dawant, S.L. Hartmann, and S. Gadamsetty. Brain atlas deformation in the presence of large space-occupying tumors. In MICCAI 99, pages 589-596, 1999. 